\title{
Analysis of multi-stage open shop processing systems*
}

\author{
Christian E.J. Eggermont ${ }^{1}$, Alexander Schrijver², and Gerhard J. \\ Woeginger $^{1}$
}

1 Department of Mathematics and Computer Science

TU Eindhoven, The Netherlands

c.e.j.eggermont@tue.nl; gwoegi@win.tue.nl

2 CWI and University of Amsterdam, Netherlands

lex@cwi.nl

\begin{abstract}
We study algorithmic problems in multi-stage open shop processing systems that are centered around reachability and deadlock detection questions.

We characterize safe and unsafe system states. We show that it is easy to recognize system states that can be reached from the initial state (where the system is empty), but that in general it is hard to decide whether one given system state is reachable from another given system state. We show that the problem of identifying reachable deadlock states is hard in general open shop systems, but is easy in the special case where no job needs processing on more than two machines (by linear programming and matching theory), and in the special case where all machines have capacity one (by graph-theoretic arguments).
\end{abstract}

1998 ACM Subject Classification F.2.2

Keywords and phrases Scheduling; resource allocation; deadlock; computational complexity

Digital Object Identifier 10.4230/LIPIcs.STACS.2011.484

\section{Introduction}

We consider a multi-stage open shop processing system with $n$ jobs $J_{1}, \ldots, J_{n}$ and $m$ machines $M_{1}, \ldots, M_{m}$. Every job $J_{j}(j=1, \ldots, n)$ requests processing on a certain subset $\mathcal{M}\left(J_{j}\right)$ of the machines; the ordering in which job $J_{j}$ passes through the machines in $\mathcal{M}\left(J_{j}\right)$ is irrelevant and can be chosen arbitrarily by the scheduler. Every machine $M_{i}(i=1, \ldots, m)$ has a corresponding capacity $\operatorname{cap}\left(M_{i}\right)$, which means that at any moment in time it can simultaneously hold and process up to $\operatorname{cap}\left(M_{i}\right)$ jobs. For more information on multi-stage scheduling systems, the reader is referred to the survey [6].

In this article, we are mainly interested in the performance of real-time multi-stage systems, where the processing time $p_{j, i}$ of job $J_{j}$ on machine $M_{i}$ is a priori unknown and hard to predict. The Central Control (the scheduling policy) of the system learns the processing time $p_{j, i}$ only when the processing of job $J_{j}$ on machine $M_{i}$ is completed. The various jobs move through the system in an unsynchronized fashion. Here is the standard behavior of a job in such a system:

* This research has been supported by the Netherlands Organisation for Scientific Research (NWO), grant 639.033.403; by DIAMANT (an NWO mathematics cluster); by the Future and Emerging Technologies unit of the European Community (IST priority), under contract no. FP6-021235-2 (project ARRIVAL); by BSIK grant 03018 (BRICKS: Basic Research in Informatics for Creating the Knowledge Society).

(c) (i) () $($ ) Christian E.J. Eggermont, Alexander Schrijver, Gerhard J. Woeginger;

28th Symposium on Theoretical Aspects of Computer Science (STACS'11).

Editors: Thomas Schwentick, Christoph Dürr; pp. 484-494

Leibniz International Proceedings in Informatics



LI I ICS Schloss Dagstuhl - Leibniz-Zentrum für Informatik, Dagstuhl Publishing, Germany 
1. In the beginning the job is asleep and is waiting outside the system. For technical reasons, we assume that the job occupies an artificial machine $M_{0}$ of unbounded capacity.

2. After a finite amount of time the job wakes up, and starts looking for an available machine $M$ on which it still needs processing. If the job detects such a machine $M$, it requests permission from the Central Control to move to machine $M$. If no such machine is available or if the CENTRAL CONTROL denies permission, the job falls asleep again (and returns to the beginning of Step 2).

3. If the job receives permission to move, it releases its current machine and starts processing on the new machine $M$. While the job is being processed and while the job is asleep, it continuously occupies machine $M$ (and blocks one of the $\operatorname{cap}(M)$ available places on $M)$. When the processing of the job on machine $M$ is completed and in case the job still needs processing on another machine, it returns to Step 2.

4. As soon as the processing of the job on all relevant machines is completed, the job informs the Central Control that it is leaving the system. We assume that the job then moves to an artificial final machine $M_{m+1}$ (with unbounded capacity), and disappears.

The described system behavior typically occurs in robotic cells and flexible manufacturing systems. The high level goal of the Central Control is to arrive at the situation where all the jobs have been completed and left the system. Other goals are of course to reach a high system throughput, and to avoid unnecessary waiting times of the jobs. However special care has to be taken to prevent the system from reaching situations of the following type:

- Example 1. Consider an open shop system with three machines $M_{1}, M_{2}, M_{3}$ of capacity 1 . There are three jobs that each require processing on all three machines. Suppose that the Central Control behaves as follows:

The first job requests permission to move to machine $M_{1}$. Permission granted.

The second job requests permission to move to machine $M_{2}$. Permission granted.

The third job requests permission to move to machine $M_{3}$. Permission granted.

Once the three jobs have completed their processing on theses machines, they keep blocking their machines and simultaneously keep waiting for the other machines to become idle. The processing never terminates.

Example 1 illustrates a so-called deadlock, that is, a situation in which the system gets stuck and comes to a halt since no further processing is possible: Every job in the system is waiting for resources that are blocked by other jobs that are also waiting in the system. Resolving a deadlock is usually expensive (with respect to time, energy, and resources), and harmfully diminishes the system performance. In robotic cells resolving a deadlock typically requires human interaction. The scientific literature on deadlocks is vast, and touches many different areas like flexible manufacturing, automated production, operating systems, Petri nets, network routing, etc.

The literature distinguishes two basic types of system states (see for instance Coffman, Elphick \& Shoshani [2], Gold [5], or Banaszak \& Krogh [1]). A state is called safe, if there is at least one possible way of completing all jobs. A state is called unsafe, if every possible continuation eventually will get stuck in a deadlock. An example for a safe state is the initial situation where all jobs are outside the system (note that the jobs could move sequentially through the system and complete). Another example for a safe state is the final situation where all jobs have been completed. An example for an unsafe state are the deadlock states. 


\section{Summary of considered problems and derived results}

In this article we study the behavior of safe and unsafe states in open shop scheduling systems. In particular, we investigate the computational complexity of the four algorithmic questions described in the following paragraphs. First, if one wants to have a smoothly running system, then it is essential to distinguish the safe from the unsafe system states:

\section{Problem: Safe State Recognition}

InSTANCE: An open shop scheduling system. A system state $s$.

Question: Is state $s$ safe?

Section 3 provides a simple characterization of unsafe states, which leads to a (straightforward) polynomial time algorithm for telling safe states from unsafe states. Similar characterizations have already been given a decade ago in the work of Sulistyono \& Lawley [9] and Xing, Lin \& $\mathrm{Hu}[10]$. Our new argument is extremely short and simple.

One of the most basic problems in analyzing a system consists in characterizing those system states that can be reached while the shop is running.

\section{Problem: Reachable State Recognition}

InSTANCE: An open shop scheduling system. A system state $s$.

Question: Can the system reach state $s$ when starting from the initial situation where all machines are still empty?

In Section 4 we derive a polynomial time algorithm for recognizing reachable system states. The main idea is to reverse the time axis, and to make the system run backward. Then reachable states in the original system translate into safe states in the reversed system, and the results from Section 3 can be applied.

Hence recognizing states that are reachable from the initial situation is easy. What about recognizing states that are reachable from some other given state?

\section{Problem: State-to-State Reachability}

Instance: An open shop scheduling system. Two system states $s$ and $t$.

QUESTION: Can the system reach state $t$ when starting from state $s$ ?

Surprisingly, there is a strong and sudden jump in the computational complexity of the reachability problem: Section 5 provides an NP-hardness proof for problem STATE-TOState ReACHABILITY.

Another fundamental question is whether an open shop system can ever fall into a deadlock. In case it cannot, then there are no reachable unsafe states and the CENTRAL CONTROL may permit all moves right away and without analyzing them; in other words the system is fool-proof and will run smoothly without supervision.

\section{Problem: Reachable DeAdlock}

INSTANCE: An open shop scheduling system.

QUESTION: Can the system ever reach a deadlock state when starting from the initial situation?

Section 6 proves problem REACHABLE DEADLOCK to be NP-hard, even for the highly restricted special case where the capacity of each machine is at most three and where each job requires processing on at most four machines. In Sections 7 and 8 we exhibit two special 
cases for which this problem is solvable in polynomial time: The special case where every job needs processing on at most two machines is settled by a linear programming formulation and techniques from matching theory. The special case where every machine has capacity one is solved by analyzing cycles in certain edge-colored graphs.

Because of the page limit, some of the proofs are missing and will only appear in the full version of the paper.

\section{Basic definitions}

A state of an open shop scheduling system is a snapshot describing a situation that might potentially occur while the system is running. A state $s$ specifies for every job $J_{j}$

- the machine $M^{s}\left(J_{j}\right)$ on which this job is currently waiting or currently being processed,

- and the set $\mathcal{M}^{s}\left(J_{j}\right) \subseteq \mathcal{M}\left(J_{j}\right)-\left\{M^{s}\left(J_{j}\right)\right\}$ of machines on which the job still needs future processing.

The machines $M^{s}\left(J_{j}\right)$ implicitly determine

- the set $\mathcal{J}^{s}\left(M_{i}\right) \subseteq\left\{J_{1}, \ldots, J_{n}\right\}$ of jobs currently handled by machine $M_{i}$.

The initial state 0 is the state where all jobs are still waiting for their first processing; in other words in the initial state all jobs $J_{j}$ satisfy $M^{0}\left(J_{j}\right)=M_{0}$ and $\mathcal{M}^{0}\left(J_{j}\right)=\mathcal{M}\left(J_{j}\right)$. The final state $f$ is the state where all jobs have been completed; in other words in the final state all jobs $J_{j}$ satisfy $M^{f}\left(J_{j}\right)=M_{m+1}$ and $\mathcal{M}^{f}\left(J_{j}\right)=\emptyset$.

A state $t$ is called a successor of a state $s$, if it results from $s$ by moving a single job $J_{j}$ from its current machine $M^{s}\left(J_{j}\right)$ to some new machine in set $\mathcal{M}^{s}\left(J_{j}\right)$, or by moving a job $J_{j}$ with $\mathcal{M}^{s}\left(J_{j}\right)=\emptyset$ from its current machine to $M_{m+1}$. In this case we will also say that the system moves from $s$ to $t$. This successor relation is denoted $s \rightarrow t$. A state $t$ is said to be reachable from state $s$, if there exists a finite sequence $s=s_{0}, s_{1}, \ldots, s_{k}=t$ of states (with $k \geq 0$ ) such that $s_{i-1} \rightarrow s_{i}$ holds for $i=1, \ldots, k$. A state $s$ is called reachable, if it is reachable from the initial state 0 .

- Lemma 2. Any reachable state $s$ can be reached from the initial state through a sequence of at most $n+\sum_{i=1}^{n}\left|\mathcal{M}\left(J_{j}\right)\right|$ moves.

A state is called safe, if the final state $f$ is reachable from it; otherwise the state is called unsafe. A state is a deadlock, if it has no successor states and if it is not the final state $f$.

\section{Analysis of unsafe states}

Unsafe states in open shop systems are fairly well-understood, and the literature contains several characterizations for them; see for instance Sulistyono \& Lawley [9], Xing, Lin \& Hu [10], and Lawley [7]. In this section we provide yet another analysis of unsafe states, which is shorter and (as we think) simpler than the previously published arguments.

A machine $M$ is called full in state $s$, if it is handling exactly cap $(M)$ jobs. A non-empty subset $\mathcal{B}$ of the machines is called blocking for state $s$,

- if every machine in $\mathcal{B}$ is full, and

- if every job $J_{j}$ that occupies some machine in $\mathcal{B}$ satisfies $\emptyset \neq \mathcal{M}^{s}\left(J_{j}\right) \subseteq \mathcal{B}$.

Here is a simple procedure that determines whether a given machine $M_{i}$ is part of a blocking set in state $s$ : Let $\mathcal{B}_{0}=\left\{M_{i}\right\}$. For $k \geq 1$ let $\mathcal{J}_{k}$ be the union of all job sets $\mathcal{J}^{s}(M)$ with $M \in \mathcal{B}_{k-1}$, and let $\mathcal{B}_{k}$ be the union of all machine sets $\mathcal{M}^{s}(J)$ with $J \in \mathcal{J}_{k}$. Clearly $\mathcal{B}_{0} \subseteq \mathcal{B}_{1} \subseteq \cdots \subseteq \mathcal{B}_{m-1}=\mathcal{B}_{m}$. Furthermore machine $M_{i}$ belongs to a blocking set, if and only if $\mathcal{B}_{m}$ is a blocking set, if and only if all machines in $\mathcal{B}_{m}$ are full. In case $\mathcal{B}_{m}$ is a 
blocking set, we denote it by $\mathcal{B}_{\text {min }}^{s}\left(M_{i}\right)$ and call it the canonical blocking set for machine $M_{i}$ in state $s$. The canonical blocking set is the smallest blocking set containing $M_{i}$ :

- Lemma 3. If machine $M_{i}$ belongs to a blocking set $\mathcal{B}$ in state $s$, then $\mathcal{B}_{\text {min }}^{s}\left(M_{i}\right) \subseteq \mathcal{B}$.

The machines in a blocking set $\mathcal{B}$ all operate at full capacity on jobs that in the future only want to move to other machines in $\mathcal{B}$. Since these jobs are permanently blocked from moving, the state $s$ must eventually lead to a deadlock and hence is unsafe. The following theorem shows that actually every deadlock is caused by such blocking sets.

- Theorem 4. A state $s$ is unsafe if and only if it has a blocking set of machines.

Proof. The if-statement is obvious. For the only-if-statement, we classify the unsafe states with respect to their distances to deadlock states. The set $\mathcal{U}_{0}$ contains the deadlock states. For $d \geq 1$, set $\mathcal{U}_{d}$ contains all states whose successor states are all contained in $\mathcal{U}_{d-1}$. Note that $\mathcal{U}_{d-1} \subseteq \mathcal{U}_{d}$, and note that every unsafe state occurs in some $\mathcal{U}_{d}$. We prove by induction on $d$ that every state in $\mathcal{U}_{d}$ has a blocking set of machines. For $d=0$ this is trivial.

In the inductive step, assume for the sake of contradiction that some state $s \in \mathcal{U}_{d}$ is unsafe but does not contain any blocking set. Since every move from $s$ leads to a state in $\mathcal{U}_{d-1}$, all successor states of $s$ must contain blocking sets. Whenever in state $s$ some job $J$ moves to some (non-full) machine $M$, this machine $M$ must become full and must then be part of any blocking set. Among all possible moves, consider a move that yields a state $t$ with a newly full machine $M$ for which the canonical blocking set $\mathcal{B}_{\min }^{t}(M)$ is of the smallest possible cardinality.

Note that in state $t$ there exist a machine $M^{\prime} \in \mathcal{B}_{\min }^{t}(M)$ and a job $J^{\prime} \in \mathcal{J}^{t}\left(M^{\prime}\right)$ with $M \in \mathcal{M}^{t}\left(J^{\prime}\right)$; otherwise $\mathcal{B}_{\text {min }}^{t}(M)-\{M\}$ would be a blocking set for state $s$. Now consider the successor state $u$ of $s$ that results by moving job $J^{\prime}$ from machine $M$ to $M^{\prime}$. Since $\mathcal{M}^{u}\left(J^{\prime}\right) \subseteq \mathcal{B}_{\text {min }}^{t}(M)$, a simple inductive argument shows that $\mathcal{B}_{\text {min }}^{u}(M) \subseteq \mathcal{B}_{\text {min }}^{t}(M)$. Since job $J^{\prime}$ has just jumped away from $M^{\prime}$, this machine cannot be full in state $u$, and hence $M^{\prime} \in \mathcal{B}_{\min }^{t}(M)-\mathcal{B}_{\min }^{u}(M)$. Consequently the canonical blocking set $\mathcal{B}_{\min }^{u}(M)$ has smaller cardinality than $\mathcal{B}_{\min }^{t}(M)$. This contradiction completes the proof.

- Lemma 5. For a given state $s$, it can be decided in polynomial time whether $s$ has a blocking set of machines. Consequently, problem SAFE State ReCognition can be decided in polynomial time.

Proof. Create an auxiliary digraph that corresponds to state $s$ : the vertices are the machines $M_{1}, \ldots, M_{m}$. Whenever some job $J_{j}$ occupies a machine $M_{i}$, the digraph contains an arc from $M_{i}$ to every machine in $\mathcal{M}^{s}\left(J_{j}\right)$. Obviously state $s$ has a blocking set of machines if and only if the auxiliary digraph contains a strongly connected component with the following two properties: (i) All vertices in the component are full. (ii) There are no arcs leaving the component. Since the strongly connected components of a digraph can easily be determined and analyzed in linear time (see for instance [3]), the desired statement follows.

\section{$4 \quad$ Analysis of reachable states}

In this section we discuss the behavior of reachable system states. We say that a state $t$ is subset-reachable from state $s$, if every job $J_{j}$ satisfies one of the following three conditions:

- $M^{t}\left(J_{j}\right)=M^{s}\left(J_{j}\right)$ and $\mathcal{M}^{t}\left(J_{j}\right)=\mathcal{M}^{s}\left(J_{j}\right)$, or

- $M^{t}\left(J_{j}\right) \in \mathcal{M}^{s}\left(J_{j}\right)$ and $\mathcal{M}^{t}\left(J_{j}\right) \subseteq \mathcal{M}^{s}\left(J_{j}\right)-\left\{M^{t}\left(J_{j}\right)\right\}$, or

- $M^{t}\left(J_{j}\right)=M_{m+1}$ and $\mathcal{M}^{t}\left(J_{j}\right)=\emptyset$. 
Clearly whenever a state $t$ is reachable from some state $s$, then $t$ is also subset-reachable from $s$. The following example demonstrates that the reverse implication is not necessarily true. This example also indicates that the algorithmic problem REACHABLE STATE RECOGNITION (as formulated in the introduction) is not completely straightforward.

- Example 6. Consider an open shop system with two machines $M_{1}, M_{2}$ of capacity 1 and two jobs $J_{1}, J_{2}$ with $\mathcal{M}\left(J_{1}\right)=\mathcal{M}\left(J_{2}\right)=\left\{M_{1}, M_{2}\right\}$. Consider the state $s$ where $J_{1}$ is being processed on $M_{1}$ and $J_{2}$ is being processed on $M_{2}$, and where $\mathcal{M}^{s}\left(J_{1}\right)=\mathcal{M}^{s}\left(J_{2}\right)=\emptyset$. It can be seen that $s$ is subset-reachable from the initial state 0 , whereas $s$ is not reachable from 0 .

Our next goal is to derive a polynomial time algorithm for recognizing reachable system states. Consider an open shop scheduling system and a fixed system state $s$. Without loss of generality we assume that $s$ is subset-reachable from the initial state. We define a new (artificial) state $t$ where $M^{t}\left(J_{j}\right):=M^{s}\left(J_{j}\right)$ and $\mathcal{M}^{t}\left(J_{j}\right):=\mathcal{M}\left(J_{j}\right)-\mathcal{M}^{s}\left(J_{j}\right)-\left\{M^{s}\left(J_{j}\right)\right\}$ for all jobs $J_{j}$. Note that in both states $s$ and $t$ every job is sitting on the very same machine, but the work that has already been performed in state $s$ is exactly the work that still needs to be done in state $t$.

Lemma 7. State $s$ is reachable if and only if state $t$ is safe.

Proof. First assume that $s$ is reachable, and let $0=s_{0} \rightarrow s_{1} \rightarrow \cdots \rightarrow s_{k}=s$ denote a corresponding witness sequence of moves. Define a new sequence $t=t_{k} \rightarrow t_{k-1} \rightarrow \cdots \rightarrow t_{0}=f$ of moves: Whenever the move $s_{\ell} \rightarrow s_{\ell+1}(0 \leq \ell \leq k-1)$ results from moving job $J_{j}$ from machine $M_{a}$ to machine $M_{b}$, then the move $t_{\ell+1} \rightarrow t_{\ell}$ results from moving job $J_{j}$ from machine $M_{b}$ to machine $M_{a}$. (Note that the artificial machines $M_{0}$ and $M_{m+1}$ switch their roles.) Hence $t$ is safe. A symmetric argument shows that if $t$ is safe then $s$ is reachable.

Hence deciding reachability is algorithmically equivalent to deciding safeness. Together with Lemma 5 this yields the following theorem.

Theorem 8. ReAchable State Recognition can be decided in polynomial time.

The following lemma states a simple sufficient condition that makes a state reachable.

$\checkmark$ Lemma 9. Let $s$ be a state, and let $\mathcal{K}$ be a subset of machines such that every job that still needs further processing in s satisfies $M^{s}\left(J_{j}\right) \in \mathcal{K}$ and

$$
\mathcal{M}^{s}\left(J_{j}\right) \cup\left\{M^{s}\left(J_{j}\right)\right\}=\mathcal{K} \cap \mathcal{M}\left(J_{j}\right) .
$$

Then $s$ is a reachable system state.

Proof. By renaming the jobs we assume that the jobs $J_{j}$ with $1 \leq j \leq k$ have $M^{s}\left(J_{j}\right)=$ $M_{m+1}$ and the jobs $J_{j}$ with $k+1 \leq j \leq n$ have $M^{s}\left(J_{j}\right) \in \mathcal{K}$. We handle the jobs one by one in their natural order: every job moves through all machines in $\mathcal{M}\left(J_{j}\right)-\mathcal{M}^{s}\left(J_{j}\right)$, and ends up on machine $M^{s}\left(J_{j}\right)$. Then the next job is handled.

\section{Analysis of state-to-state reachability}

We establish NP-hardness of STATE-TO-STATE REACHABILITY by means of a reduction from the following satisfiability problem; see Garey \& Johnson [4]. 


\section{Problem: Three-SATisfiability}

InPUT: A set $X=\left\{x_{1}, \ldots, x_{n}\right\}$ of $n$ logical variables; a set $C=\left\{c_{1}, \ldots, c_{m}\right\}$ of $m$ clauses over $X$ that each contain three literals.

QUestion: Is there a truth assignment for $X$ that satisfies all clauses in $C$ ?

We start from an instance of ThreE-SATisfiability, and construct a corresponding instance of State-To-State Reachability for it. Throughout we will use $\ell_{i}$ to denote the unnegated literal $x_{i}$ or the negated literal $\overline{x_{i}}$ for some fixed variable $x_{i} \in X$, and we will use $\ell$ to denote a generic literal over $X$. Altogether there are $5 n+m$ machines:

- For every literal $\ell_{i}$, there are three corresponding machines $S\left(\ell_{i}\right), T\left(\ell_{i}\right)$, and $U\left(\ell_{i}\right)$. Machine $U\left(\ell_{i}\right)$ has capacity 2, whereas machines $S\left(\ell_{i}\right)$ and $T\left(\ell_{i}\right)$ have capacity 1 . For every variable $x_{i} \in X$ the two machines $U\left(x_{i}\right)$ and $U\left(\overline{x_{i}}\right)$ coincide, and the corresponding machine will sometimes simply be called $U(i)$.

- For every clause $c_{j} \in C$, there is a corresponding machine $V\left(c_{j}\right)$ with capacity 3 .

Furthermore the scheduling instance contains $4 n$ jobs that correspond to literals and $6 m$ jobs that correspond to clauses. For every literal $\ell_{i}$ there are two corresponding jobs:

- Job $J\left(\ell_{i}\right)$ is sitting on machine $S\left(\ell_{i}\right)$ in state $s$. In state $t$ it has moved to machine $U\left(\ell_{i}\right)$ without visiting other machines inbetween.

- Job $J^{\prime}\left(\ell_{i}\right)$ is still waiting outside the system in state $s$, and has already left the system in state $t$. Inbetween the job visits machines $S\left(\ell_{i}\right), T\left(\ell_{i}\right), U\left(\ell_{i}\right)$ in arbitrary order.

Consider a clause $c_{j}$ that consists of three literals $\ell_{a}, \ell_{b}, \ell_{c}$. Then the following six jobs correspond to clause $c_{j}$ :

- For $\ell \in\left\{\ell_{a}, \ell_{b}, \ell_{c}\right\}$ there is a job $K\left(c_{j}, \ell\right)$ that in state $s$ sits on machine $V\left(c_{j}\right)$, then moves through machines $S(\ell)$ and $T(\ell)$ in arbitrary order, and finally has left the system in state $t$. Note that in state $s$ these three jobs block machine $V\left(c_{j}\right)$ to full capacity.

- For $\ell \in\left\{\ell_{a}, \ell_{b}, \ell_{c}\right\}$ there is another job $K^{\prime}\left(c_{j}, \ell\right)$ that waits outside the system in state $s$, then moves through machines $U(\ell)$ and $V\left(c_{j}\right)$ in arbitrary order, and finally has left the system in state $t$.

In the full version of the paper, we will show that in the constructed scheduling instance state $t$ is reachable from state $s$ if and only if the THREE-SATISFIABILITY instance has a satisfying truth assignment. This then implies the following theorem.

- Theorem 10. State-To-State Reachability is NP-complete.

\section{Analysis of reachable deadlocks}

In this section we show that Reachable DeADlOCK is an NP-hard problem. Our reduction is from the following variant of the Three-Dimensional Matching problem; see Garey \& Johnson [4, p.221].

Problem: Three-Dimensional Matching

INSTANCE: An integer $n$. Three pairwise disjoint sets $A=\left\{a_{1}, \ldots, a_{n}\right\}, B=$ $\left\{b_{1}, \ldots, b_{n}\right\}$, and $C=\left\{c_{1}, \ldots, c_{n}\right\}$. A set $T \subseteq A \times B \times C$ of triples, such that every element occurs in at most three triples in $T$.

QUESTION: Does there exist a subset $T^{\prime} \subseteq T$ of $n$ triples, such that every element in $A \cup B \cup C$ occurs in exactly one triple in $T^{\prime}$ ?

We start from an arbitrary instance of THREE-DimENSIONAL MATCHING, and construct the following corresponding instance of Reachable Deadlock for it. There are two types of machines. Note that every machine has capacity at most three. 
- There are $n+2$ so-called structure machines $S_{0}, \ldots, S_{n+1}$, each of capacity 1 .

- For every triple $t \in T$, there is a corresponding triple machine $T_{t}$ with capacity 3.

Furthermore there are $4 n+2$ jobs.

- For every element $a_{i} \in A$ there are two corresponding $A$-element jobs $J^{+}\left(a_{i}\right)$ and $J^{-}\left(a_{i}\right)$. Job $J^{+}\left(a_{i}\right)$ requires processing on structure machine $S_{i}$, and on every triple machine $T_{t}$ with $a_{i} \in t$. Job $J^{-}\left(a_{i}\right)$ requires processing on structure machine $S_{i-1}$, and on every triple machine $T_{t}$ with $a_{i} \in t$.

- For every element $b_{i} \in B$ there is a corresponding $B$-element job $J\left(b_{i}\right)$ that requires processing on structure machine $S_{n+1}$, and on every triple machine $T_{t}$ with $b_{i} \in t$.

- For every element $c_{i} \in C$ there is a corresponding $C$-element job $J\left(c_{i}\right)$ that requires processing on structure machine $S_{n+1}$, and on every triple machine $T_{t}$ with $c_{i} \in t$.

- Finally there is a dummy job $D_{0}$ that needs processing on $S_{0}$ and $S_{n+1}$, and another dummy job $D_{n+1}$ that needs processing on $S_{n}$ and $S_{n+1}$.

Since every element of $A \cup B \cup C$ occurs in at most three triples, we note that each job requires processing on at most four machines. For the ease of later reference, we also list for every machine the jobs that need processing on that machine.

- A triple machine $T_{t}$ with $t=\left(a_{i}, b_{j}, c_{k}\right)$ handles the four jobs $J^{+}\left(a_{i}\right), J^{-}\left(a_{i}\right), J\left(b_{j}\right)$, and $J\left(c_{k}\right)$.

- Structure machine $S_{i}$ with $1 \leq i \leq n-1$ handles the jobs $J^{+}\left(a_{i}\right)$ and $J^{-}\left(a_{i+1}\right)$.

Structure machine $S_{0}$ handles the two jobs $J^{-}\left(a_{1}\right)$ and $D_{0}$.

Structure machine $S_{n}$ handles the two jobs $J^{+}\left(a_{n}\right)$ and $D_{n+1}$.

Structure machine $S_{n+1}$ handles $2 n+2$ jobs: $D_{0}, D_{n+1}$, all B-element jobs, and all C-element jobs.

The following theorem contains the main result of this section.

- Theorem 11. REACHABLE DEADLOCK is NP-complete, even if the capacity of each machine is at most three, and if each job requires processing on at most four machines.

Indeed, Lemma 2 yields an NP-certificate for problem REACHABLE DEADLOCK. The hardness argument proves that the constructed scheduling instance has a reachable deadlock if and only if the ThreE-Dimensional MATCHing instance has answer YES. All details are provided in the full version of the paper.

\section{Reachable deadlocks if jobs require two machines}

Throughout this section we only consider open shop systems where $|\mathcal{M}(J)|=2$ holds for all jobs $J$. We introduce for every job $J$ and for every machine $M \in \mathcal{M}(J)$ a corresponding real variable $x(J, M)$, and for every machine $M$ a corresponding real variable $y(M)$. Our analysis is centered around the following linear program (LP):

$$
\begin{array}{lll}
\min & \sum_{M} \max \{y(M), \operatorname{cap}(M)\} & \\
\text { s.t. } & \sum_{J: M \in \mathcal{M}(J)} x(J, M)=y(M) & \text { for all machines } M \\
& \sum_{M \in \mathcal{M}(J)} x(J, M)=1 & \text { for all jobs } J \\
& x(J, M) \geq 0 & \text { for all } J \text { and } M \in \mathcal{M}(J)
\end{array}
$$

Although this linear program is totally unimodular, we will mainly deal with its fractional solutions. 
- Lemma 12. One can compute in polynomial time an optimal solution for the linear program (LP) that additionally satisfies the following property $\left({ }^{*}\right)$ for every job $J$ with $\mathcal{M}(J)=\left\{M_{a}, M_{b}\right\}$ : If $y\left(M_{a}\right) \geq \operatorname{cap}\left(M_{a}\right)$ and $x\left(J, M_{a}\right)>0$, then $y\left(M_{b}\right) \geq \operatorname{cap}\left(M_{b}\right)$.

Proof. We determine in polynomial time an optimal solution of (LP). Then we perform a polynomial number of post-processing steps on this optimal solution, as long as there exists a job violating property $\left(^{*}\right)$. In this case $y\left(M_{a}\right) \geq \operatorname{cap}\left(M_{a}\right), x\left(J, M_{a}\right)>0$, and $y\left(M_{b}\right)<\operatorname{cap}\left(M_{b}\right)$.

The post-processing step decreases the values $x\left(J, M_{a}\right)$ and $y\left(M_{a}\right)$ by some $\varepsilon>0$, and simultaneously increases $x\left(J, M_{b}\right)$ and $y\left(M_{b}\right)$ by the same $\varepsilon$. By picking $\varepsilon$ smaller than the minimum of $\operatorname{cap}\left(M_{b}\right)-y\left(M_{b}\right)$ and $x\left(J, M_{a}\right)$ this will yield another feasible solution for (LP). What happens to the objective value? If $y\left(M_{a}\right)>\operatorname{cap}\left(M_{a}\right)$ at the beginning of the step, then the step would decrease the objective value, which contradicts optimality. If $y\left(M_{a}\right)=$ $\operatorname{cap}\left(M_{a}\right)$ at the beginning of the step, then the step leaves the objective value unchanged, and yields another optimal solution with $y\left(M_{a}\right)<\operatorname{cap}\left(M_{a}\right)$ and $y\left(M_{b}\right)<\operatorname{cap}\left(M_{b}\right)$.

To summarize, every post-processing step decreases the number of machines $M$ with $y(M)=\operatorname{cap}(M)$. Hence the entire procedure terminates after at most $m$ steps.

Let $x^{*}(J, M)$ and $y^{*}(M)$ denote an optimal solution of (LP) that satisfies the property $\left.{ }^{*}\right)$ in Lemma 12. Let $\mathcal{M}^{*}$ be the set of machines $M$ with $y^{*}(M) \geq \operatorname{cap}(M)$.

- Lemma 13. The open shop system has a reachable deadlock, if and only if $\mathcal{M}^{*} \neq \emptyset$.

Proof. (Only if). Consider a reachable deadlock state, let $\mathcal{B}^{\prime}$ be the corresponding blocking set of machines, and let $\mathcal{J}^{\prime}$ be the set of jobs waiting on these machines. Every job $J \in \mathcal{J}^{\prime}$ is sitting on some machine in $\mathcal{B}^{\prime}$, and is waiting for some other machine in $\mathcal{B}^{\prime}$. Since $|\mathcal{M}(J)|=2$, this implies $\mathcal{M}(J) \subseteq \mathcal{B}^{\prime}$ for every job $J \in \mathcal{J}^{\prime}$. Then

$$
\sum_{M \in \mathcal{B}^{\prime}} y^{*}(M) \geq \sum_{J \in \mathcal{J}^{\prime}} \sum_{M \in \mathcal{M}(J)} x^{*}(J, M)=\left|\mathcal{J}^{\prime}\right| .
$$

Since furthermore $\left|\mathcal{J}^{\prime}\right|=\sum_{M \in \mathcal{B}^{\prime}} \operatorname{cap}(M)$, we conclude $y^{*}(M) \geq \operatorname{cap}(M)$ for at least one machine $M \in \mathcal{B}^{\prime}$.

(If). Let $\mathcal{J}^{*}$ be the set of jobs with $x^{*}(J, M)>0$ for some $M \in \mathcal{M}^{*}$. Property (*) in Lemma 12 now yields the following for every job $J$ : If $J \in \mathcal{J}^{*}$, then $\mathcal{M}(J) \subseteq \mathcal{M}^{*}$. Construct a bipartite graph $G$ between the jobs in $\mathcal{J}^{*}$ and the machines in $\mathcal{M}^{*}$, with an edge between $J$ and $M$ if and only if $M \in \mathcal{M}(J)$. For any subset $\mathcal{M}^{\prime} \subseteq \mathcal{M}^{*}$, the number of job neighbors in this bipartite graph is at least $\sum_{M \in \mathcal{M}^{\prime}} y^{*}(M) \geq \sum_{M \in \mathcal{M}^{\prime}} \operatorname{cap}(M)$. A variant of Hall's theorem from matching theory [8] now yields that there exists an assignment of some jobs from $\mathcal{J}^{*}$ to machines in $\mathcal{M}^{*}$ such that every $M \in \mathcal{M}^{*}$ receives cap $(M)$ pairwise distinct jobs.

To reach a deadlock, we first send all non-assigned jobs one by one through the system. They are completed and disappear. Then the assigned jobs enter the system, each moving straightly to the machine to which it has been assigned. Then the system falls into a deadlock with blocking set $\mathcal{M}^{*}$ : All machines in $\mathcal{M}^{*}$ are full, and all jobs are only waiting for machines in $\mathcal{M}^{*}$.

Since jobs $J$ with $|\mathcal{M}(J)|=1$ are harmless and may be disregarded with respect to deadlocks, we arrive at the following theorem.

- Theorem 14. For open shop systems where each job requires processing on at most two machines, Reachable Deadlock can be solved in polynomial time. 
The following example illustrates that the above LP-based approach cannot be carried over to the case where every job requires processing on three machines (since the only-if part of Lemma 13 breaks down).

- Example 15. Consider a system with two jobs and four machines of unit capacity. Job $J_{1}$ needs processing on $M_{1}, M_{2}, M_{3}$, and job $J_{2}$ needs processing on $M_{1}, M_{2}, M_{4}$. A (reachable) deadlock results if $J_{1}$ enters the system on $M_{3}$ and then moves to $M_{1}$, whereas $J_{2}$ simultaneously enters the system on $M_{4}$ and then moves to $M_{2}$.

We consider a feasible solution with $x(J, M) \equiv 1 / 3$ for every $J$ and every $M \in \mathcal{M}(J)$, and $y\left(M_{1}\right)=y\left(M_{2}\right)=2 / 3$ and $y\left(M_{3}\right)=y\left(M_{4}\right)=1 / 3$. The objective value is 4 , and hence this is an optimal solution. The post-processing leaves the solution untouched, and the resulting set $\mathcal{M}^{*}$ is empty.

\section{Reachable deadlocks if machines have unit capacity}

Throughout this section we only consider open shop systems with $\operatorname{cap}\left(M_{i}\right) \equiv 1$. For each such system we define a corresponding undirected edge-colored multi-graph $G=(V, E)$ : The vertices are the machines $M_{1}, \ldots, M_{m}$. Every job $J_{j}$ induces a clique of edges on the vertex set $\mathcal{M}\left(J_{j}\right)$, and all these edges receive color $c_{j}$. Intuitively, if two machines are connected by an edge $e$ of color $c_{j}$, then job $J_{j}$ may move between these machines along edge $e$.

- Lemma 16. For an open shop system with unit machine capacities and its corresponding edge-colored multi-graph the following two statements are equivalent.

(i) The multi-graph contains a simple cycle whose edges have pairwise distinct colors.

(ii) The system can reach a deadlock.

Proof. Assume that (i) holds, and consider a simple cycle $C$ whose edges have pairwise distinct colors. By renaming jobs and machines we may assume that the vertices in $C$ are the machines $M_{1}, \ldots, M_{k}$, and that the edges in $C$ are $\left[M_{j}, M_{j+1}\right]$ with color $c_{j}$ for $1 \leq j \leq k-1$, and $\left[M_{k}, M_{1}\right]$ with colors $c_{k}$. Consider the following processing order of the jobs:

- In the first phase, the jobs $J_{j}$ with $k+1 \leq j \leq n$ are processed one by one: Job $J_{j+1}$ only enters the system after job $J_{j}$ has completed all its processing and has already left the system. At the end of this phase we are left with the jobs $J_{1}, \ldots, J_{k}$.

- In the second phase, the jobs $J_{1}, \ldots, J_{k}$ are handled one by one. When job $J_{j}$ is handled, first all operations of $J_{j}$ on machines $M_{i}$ with $i \geq k+1$ are processed. Then job $J_{j}$ moves to machine $M_{j}$, and stays there till the end of the second phase. Then the next job is handled.

At the end of the second phase, for $1 \leq i \leq k$ job $J_{i}$ is blocking machine $M_{i}$, and waiting for future processing on some other machine in cycle $C$. The system has fallen into a deadlock, and hence (i) implies (ii).

Next assume that (ii) holds, and consider a deadlock state. For every waiting job $J_{j}$ in the deadlock, let $M_{j}^{\prime}$ be the machine on which $J_{j}$ is currently waiting and let $M_{j}^{\prime \prime}$ denote one of the machines for which the job is waiting. Consider the sub-graph of $G$ that for every waiting job $J_{j}$ contains the vertex $M_{j}^{\prime}$ together with an edge $\left[M_{j}^{\prime}, M_{j}^{\prime \prime}\right]$ of color $c_{j}$. This sub-graph has as many vertices as edges, and hence must contain a simple cycle; hence (ii) implies (i).

- Lemma 17. For the edge-colored multi-graph $G=(V, E)$ corresponding to some open shop system with unit machine capacities, the following three statements are equivalent. 
(i) The multi-graph contains a simple cycle whose edges have pairwise distinct colors.

(ii) The multi-graph contains a 2-vertex-connected component that spans edges of at least two different colors.

(iii) The multi-graph contains a simple cycle whose edges have at least two different colors.

Proof. We show that (i) implies (ii) implies (iii) implies (i). The implication from (i) to (ii) is straightforward.

Assume that (ii) holds, and consider a vertex $v$ in such a 2 -vertex-connected component that is incident to two edges with two distinct colors. These two edges can be connected to a simple cycle, and we get (iii).

Assume (iii), and consider the shortest cycle $C$ whose edges have at least two different colors. If two edges $\left[u, u^{\prime}\right]$ and $\left[v, v^{\prime}\right]$ on $C$ have the same color $c_{j}$, then the vertices $u, u^{\prime}, v, v^{\prime}$ are all in the machine set $\mathcal{M}\left(J_{j}\right)$ of job $J_{j}$. Hence they span a clique in color $c_{j}$, and some edges in this clique can be used to construct a shorter cycle with edges of at least two different colors. This contradiction shows that (iii) implies (i).

Lemmas 16 and 17 together yield that an open shop system can fall into a deadlock state if and only if the corresponding multi-graph contains a 2-vertex-connected component that spans edges of at least two different colors. Since the 2-vertex-connected components of a graph can easily be determined and analyzed in linear time (see for instance [3]), we arrive at the following theorem.

- Theorem 18. For open shop systems with unit machine capacities, problem REACHABLE DEADLOCK can be solved in polynomial time.

\section{References}

1 Z.A. Banaszak and B.H. Krogh (1990). Deadlock avoidance in flexible manufacturing systems with concurrently competing process flows. IEEE Transactions on Robotics and Automation 6, 724-734.

2 E.G. Coffman, M.J. Elphick, and A. Shoshani (1971). System Deadlocks. ACM Computing Surveys 3, 67-78.

3 T.H. Cormen, C.E. Leiserson, R.L. Rivest, and C. Stein (2001). Introduction to Algorithms. MIT Press.

4 M.R. Garey and D.S. Johnson (1979). Computers and Intractability: A Guide to the Theory of NP-Completeness. Freeman, San Francisco.

5 M. Gold (1978). Deadlock prediction: Easy and difficult cases. SIAM Journal on Computing 7, 320-336.

6 E.L. Lawler, J.K. Lenstra, A.H.G. Rinnooy Kan, and D.B. Shmoys (1993). Sequencing and scheduling: Algorithms and complexity. In: Handbooks in Operations Research and Management Science, Vol. 4, North Holland, 445-522.

7 M. Lawley (1999). Deadlock avoidance for production systems with flexible routing. IEEE Transactions on Robotics and Automation 15, 497-510.

8 L. Lovász and M.D. Plummer (1986). Matching Theory. Annals of Discrete Mathematics 29, North-Holland.

9 W. Sulistyono and M. Lawley (2001). Deadlock avoidance for manufacturing systems with partially ordered process plans. IEEE Transactions on Robotics and Automation 17, 819832.

10 K. Xing, F. Lin, and B. Hu (2001). An optimal deadlock avoidance policy for manufacturing system with flexible operation sequence and flexible routing. Proceedings of the 2001 IEEE International Conference on Robotics and Automation (ICRA'2001), 3565-3570. 